\title{
IoT-based Tetra Health Surveillance System (THSS)
}

\author{
Debanwita Ghosh ${ }^{1}$, Debdulal Sau ${ }^{2}$, Shubhrajit Nandy ${ }^{3}$, Meghasweta Pal ${ }^{4}$, Riju Bhattacherjee ${ }^{5}$, Rupanjana Das ${ }^{6}$, \\ Dr. Rajesh Bose ${ }^{7}$, Dr. Sandip Roy ${ }^{8}{ }^{8}$, \\ 1,2,3,4,5,6, 7, 8 Department of Computational Science, Brainware University
}

\begin{abstract}
:
Nowadays, we are very conscious about our health because of how the covid-19 virus has changed our day-to-day life. IoT healthcare devices can be really helpful in this pandemic situation when we are all stuck in our homes. We have witnessed that IoT devices got very popular during this pandemic and the term 'IoT' also got really famous in the healthcare industry. If the hospital is far away from someone's home, then using these portable monitoring devices one can keep track of their well-being. Health is the most important aspect for any individual, so in this paper, we propose a solution named the IoT-based Tetra Health Surveillance System (THSS) which helps us to monitor people who live by themselves or elderly people who want to continuously monitor their vitals due to underlying health conditions. Our proposed solution also displays the Temperature and Humidity, Blood pressure, Body Temperature, and $\mathrm{SpO} 2 / \mathrm{Blood}$ Oxygen level at any given moment. This work will have a significant technical and commercial benefit because an individual can monitor all their vitals in one place and they don't have to buy separate pieces of equipment to monitor each one of their vitals. This proposed device can be an all-in-one solution that is cost-effective and easy to use.
\end{abstract}

Keywords: Healthcare Monitoring System, Tetra Health Surveillance System, Healthcare Device, IoT based Health Monitoring Devices, Covid-19, Portable Health Monitoring Device.

\section{Introduction:}

Coronavirus has caused a pandemic around the world. It spreads easily through normal human interaction, although it is extremely contagious. Currently, advanced information technologies are opening new doors to innovation in our daily lives. Due to the increase in COVID patients, the number of doctors per patient has also decreased. Patients typically face issues such as waiting in the queue, traveling from one place to another, and waiting for doctors. By using this system, these problems can be solved.

As per WHO, individuals have a fundamental right to the best possible health. Hence, we propose an innovative system combining some useful sensors. With the help of this apparatus, a physician will be able to record vital parameters of patients and share their status with them. Due to the lack of regular monitoring by physicians, maintaining personal health is imperative in such cases [1]. Remote health monitoring technology is always a better idea in areas where the outbreak is widespread. Therefore, a health surveillance system based on the IoT is the current solution for it [2]. The hospital's operating costs can be reduced with the use of this IoT device. If the IoT device is provided to all patients to avoid wasting time and money in the hospital, the cost is as inexpensive as the benefits. IoT will have a significant impact on the 21 st century, enabling everyday devices to connect with the internet and exchange data. IoT plays a critical role in improving the quality of healthcare for patients [3].

A perfect ecosystem of smart devices will one day connect to each other to improve and simplify our lives. Devices like these can be found in many forms, such as automobiles, thermometers, micro-ovens, smart bulbs, alarm clocks, vacuum cleaners, and a variety of other devices. Here is just a partial list of IoT-based technologies. Briefly, IoT refers to the connection of devices via the internet. Based on the idea of IoT, we are designing a system called IoT Tetra Health Surveillance System (THSS).

It is possible to build a prototype board using NodeMCU firmware with open-source code. One keyword, node, and another, microcontroller, are associated with NodeMCU. The NodeMCU is an embedded microcontroller with Wi-Fi capability. Using a data cable, it can be connected to any computer with a micro USB port. You must keep in mind that the device is operated at $3.3 \mathrm{~V}$ logic level. In conclusion, you will receive a 3.3V digital high signal. Giving a 
higher voltage signal than $3.3 \mathrm{~V}$ would be unsafe for the controller, however, the input voltage can be between 5 and 12V. NodeMCU contains 16 general purpose input-output pins, 1 Analog pin, 1 Reset pin, and 1 Power pin [3].

Tetra is a prefix often used for "four". There are many scientific as well as technical terms that use it. The term tetra is used in chemistry to refer to compounds containing four atoms or groups of atoms. We call the system a "Tetra System" since it consists of four sensors, including a BP sensor, DHT-11 temperature, and humidity sensor, SpO2 sensor, and a body-temperature sensor. Patients in critical condition are monitored 24/7. Blood Pressure is an important sign. Non-invasive as well as invasive methods are available to measure Blood Pressure. Non-invasive methods do not require piercings. Blood pressure is measured using a non-invasive blood pressure sensor. With the help of the forced oscillation technique, systolic, diastolic, and mean arterial pressure is measured.

This sensor module is a calibration-based digital signal output that provides the module's humidity and temperature complexity to a specific range. By using DHT11, we can obtain accurate humidity and temperature values that are highly reliable and stable for a long time. An 8-bit microcontroller is integrated into this sensor, allowing it to measure humidity and temperature with a resistive type component and an NTC type component, providing a fast response and enabling a 4-pin single-row package for easy mounting. Serial communication is used with DHT11 sensors. Pulse rates for specific time intervals will be sent by this module. An adjusted command is needed before NodeMCU can be used to receive data. After adjusting the command, it takes about 5 - 6 milliseconds. Using the serial interface, system integration is fast and easy.

SpO2s (pulse oximeters) provide a method of monitoring oxygenation and pulse rates in a variety of situations [2]. This sensor works on the protocol of I2C communication which enables the SCL and SDA pin of the sensor to communicate with the microcontroller [4].

A body Temperature Sensor is a physical sensor that reads data through physical contact. It measures the temperature of your body by placing your finger on the sensor and it takes a couple of seconds to take input and after that, it will show you the output. This sensor provides the output in Celsius but if you need to output in Fahrenheit, then you need to manually convert this number in your code. This device is very easy to use and people from every spectrum can use this device without any hiccups.

\section{Related Work:}

The system proposed can be used for regular checkups of COVID patients while maintaining social distance. The Doctor directly gets the real-time data that is sensed by the sensor. It reduces the visiting cost of the doctor. The IoT platform used in the system helps to transfer the real-time patient data remotely to the host device. Daily health records can be maintained and can be viewed easily on graph charts for ease for doctors to see any abrupt changes in oxygen level or rise in temperature. The microcontroller acts as an LCD display that shows the patient's health condition and is connected via a wi-fi module to send the data to the webserver (wireless sensing node) [1]. The body temperature, Heart Rate with Oxygen Saturation (SpO2) of a patient can be measured with the help of this microcontroller. In the healthcare domain, IoT has a huge contribution. Smart healthcare and advanced medical resources can be obtained with the help of IoT. The obtained data can be recorded, examined, and can be clustered to predict the diseases earlier and also to help in treatment as well as to make the treatment more affordable, while also improving outcomes [2]. IoT has an enormous impact on the healthcare industry. A 24/7 health-monitoring device is needed for analyzing the patient's vital data. With the help of this device, we can get accurate monitoring data in an immediate critical situation. In the absence of the patient's caretaker, this is crucial for managing the health of old patients and children. We can check the patient's pulse rate along with the oxygen saturation and body temperature by using BPM and SpO2 sensor(MAX30100) and Temperature sensor(LM35) respectively. Sensed data is recorded and uploaded to the cloud server. Machine learning algorithms like Linear Regression are used to predict future temperature and pulse rate [3].

As per WHO statistics, cardiovascular diseases are the number one cause for the death of one out of three healthy people and these people are under the age of 75. Every year cardiovascular diseases claim almost 17 million lives worldwide. Cardiovascular diseases can cause heart attack, cardiac arrest, and even stroke. It is the second deadliest disease after cancer that claims millions of lives each year. Nowadays regular health checkup is mandatory for every human being, which poses a great impact on existing medical science. In response to the growing population and rising medical costs, the demand for smart and reliable healthcare has been increasing. A health monitoring system is required to detect the change in health conditions according to the monitored data. Many remote health care services have been inspired by mobile and cloud technologies. In our current scenario, most of our medical information gets stored in a cloud infrastructure. These medical records can be easily sent to any medical professional in the world and the patients can also keep their data/medical record with them for an extended period of time. Here, In this project, we have designed and built an E-Health Monitoring System (HMS) using an IoT platform [4].The authorities of 
healthcare are planning to provide advanced treatment in clinical organizations. Similarly, the features of Electronic Health applications and E-Health are used by many people to improve their healthcare. The SMS is submitted to the person. A health monitoring system is to be designed which monitors the symptoms like heart rate, oxygen saturation percentage, and temperature of the body in an IoT network. Internet of things is a technology that enables things around the world to connect with each other using the internet. In this technology, there will not be human intervention in connecting the things together which is a unique feature of it. The framework of IoT consists of three layers. Control layer, Device layer, and Transport layer. The Control layer is a method of security that can be used to control and manage the resources in a system. This layer comprises various devices, types of sensors, and many controllers. The transport layer will focus on communication at end-to-end providing features like high reliability, avoiding congestion, and assuring that packets will be reached in the order they were sent [5].

In recent times, remote technology has been growing to maintain different sectors. As of now, IoT has got a handle on most of the mechanical cases. Biomedical therapy is making a huge impact on our medical life like any other treatment. Medical clinics and individual health care are extended by IoT innovation [6]. Patient monitoring is not a new structure for medicinal chemistry since this technology was first applied in the year 1625 for checking the body temperature and pulse of patients. Subsequently, this framework has started to discover its utilization and acknowledgment for checking diverse sorts of physiological parameters and health-related angles that are being performed as of not long ago [7]. Nowadays the use of wireless technology is increasing for the benefit of the people and IoT has occupied most of the industrial areas, especially artificial intelligence and automation. Biomedical is one of the most trending technologies which help us improve our health. Besides hospitals, IoT device remote technology also helps and detects our personal health problems. With the help of this smart system, different kinds of parameters are detected that consume power, cost, and boost productivity [8].The world's population is growing, along with concerns over health. Thus, there is a need for health centers that are well-equipped and well-maintained. The system is used to keep records of patients' vitals, such as heart rate, body temperature, and even blood pressure. It allows us to collect patient data and the collected data will be displayed on a computer or a mobile device [9].Many elderly patients have benefited from IoT solutions for healthcare and health monitoring, allowing them to be monitored constantly throughout their lives. The impact on those living alone and their families is enormous. Health care is redefining itself through the IoT by ensuring better care, better treatment outcomes, and lower patient costs, as well as workflow improvements, improved performance, and improved patient experiences [10].

Consecutive healthcare of patients, daily visits to hospitals or clinics for health checkups, which is very unwieldy and time-taken. The IoT device enables health monitoring systems that can measure the pulse rate and body temperature of patients and show their data on a real-time basis. With the help of this device, people can control their health. Instead of going to the hospital for various health checkups, people should look for information on maintaining good health on the internet and start implementing them [11]. Every day many people are dying just for not taking care of their health and not being treated time. Also, the real-time data parameters values of our health examinations are not measured efficiently at the hospital and clinic. Sometimes it becomes difficult for the hospital to constantly examine and monitor the condition of the patient. Also, it is not possible for constant monitoring of patients in ICU. We need all these systems to prevent this type of situation. This system is mainly designed for use in hospitals to measure and monitor several frameworks like body temperature, heartbeat, room temperature and humidity, SpO2. Using NodeMCU, we can get patients' medical data and display the data on a computer or mobile device [12].

IoT is closely linked to embedded technology, with a combination of several sensors and modules connected to a smart cyst internet. The modules are connected to each other via the internet or with the help of a local network they are interconnected, which can spread to more internet worlds. With the new technology, IoT has solved many complex problems and created a new direction for smart life. From agriculture to home automation, from smart cities to healthcare, the role of IoT is unparalleled. Exploration in this sector has achieved a lot of demand lately. Using IoT in medical management has given us many benefits. First of all, it saves a lot of medical costs because these smart devices are operated remotely. It even saves valuable time for both doctors and patients, while maintaining sufficient precision [13].

Regularly, it became difficult to maintain the health maintenance and medical records of patients in hospitals. For this basis, doctors are not aware of the previous conditions of most patients. Our project is a four-sensor monitoring system known as the tetra surveillance system, which is based on IoT. Basically, in this project, our main focus is to accurately diagnose the health status of the patients and to store the medical records of the patients in a database. Smart health maintenance is an AI-based system where patient health conditions can be sensed by IOT based sensors like heart rate sensor, pulse sensor, temperature sensor, and blood pressure sensor. These sensors are connected to the NodeMCU and make a gateway, where all patient data is stored, on that basis proper treatment can be prescribed [14]. A health monitoring system is needed for the proper selection of the regular health status of patients. The result can be 
shown at any time and place, this is the main domain of the device. If any deterioration is seen in the patient's health, the doctors can be illuminated through mobile. This system is using sensors, for example, $\mathrm{SpO} 2$ sensor, Temperature sensor, and BP sensor. This system can analyze gestures of normality and abnormalities in real-time [15]. This paperwork can represent the system for observing the patient's health $24 * 7$ by using IoT. The Health monitoring system is getting much more popular among researchers and patient supervisors. This system has the ability to accurately observe physiological parameters from the patient's body every 15 seconds. This device collects the patient's vital data and sends them to the NodeMCU which is a microcontroller board via a Wi-Fi module. It helps the medical specialist to monitor the patient's health condition from time to time through their phone or desktop. As a result, this research will give appropriate and fruitful health services to the patient [16]. Using these IoT applications has made it easier for us to manage our daily lives. The IoT application plays a special role in connecting to any place, any network, any service. IoT is a progressive application and its impact on the entire human life in this present day. IoT applications use it in many more areas such as smart cities, traffic congestion, emergency services, health infrastructure, industry, and healthcare surveillance, etc. Smart cities, smart homes, and remote health surveillance are the most demandable areas of IoT applications. IoT has much more efficient applications to give increased healthcare applications such as remote health surveillance, home-based fitness health monitoring, ancient disease detection, and older people care, etc [17]. This project was designed to help solve the problem of diagnosing heart attacks at a low cost in poor countries in order to provide early treatment and to provide service to hospitals and health care centers in these countries due to the lack of access to high-cost products. It will be very difficult for the patient who lives in remote areas of the city to move to the city center for primary diagnosis. The increase in patients with heart disease and heart attacks has led to the necessity of remote heart rate monitoring, which can be used to detect emergencies and assess the risk of heart failure. A number of designers have used NodeMCU and a Wi-Fi module to implement heart rate monitors using internet of things technology (IoT) [18].

IoT has a lot of uses. Smart stop, smart house, smart city, sharp condition, mechanical spots, horticulture fields, and health monitoring are some of the uses of the Internet of Things. In medical science, IOT has such an application where a patient's well-being status can be screened and it would be more effective to check the ongoing condition of a patient. Though, all the sensors will sense the correct data of a patient, so it can easily reduce the human blunder proposed IoT-based design useful for COVID-19 disease. It helps doctors to check the health condition of the patient. It will be quite easy to take intensive care of a patient and remotely monitor the vital signs of a patient in real-time. Our system is easy to use, low-cost, and compact. This study outlines the design and development of an affordable and reliable wireless sensing device based on a NODE MCU microcontroller to collect vital signs of real-time health, such as human body temperature and heart rate. It helps in online diagnosis and managing health more efficiently, without going out of the home [19]. The advent of this technology has made it possible today to prevent deaths from various epidemics around the world. Due to the existence of different technologies, patients are able to take care of their health at the same time despite having their own busy schedules. At present patients need frequent health check-ups which are not possible in a normal medical system but it is possible with different types of tools and technologies which are available only in IoT technology. Even today, the main demand is to buy a low-cost device and accurately diagnose the disease. The usage of these services has provided better health outcomes, more satisfactory results, and costeffectiveness as compared to traditional methods [20]. The Internet has become an important part of our daily lives. It has changed people, how people will live, work, play and learn, etc. You can use the Internet for a variety of purposes including education, finance, business, entertainment, and social networking, etc. The IoT device is connected to the Internet and that type of IoT device can be controlled remotely through the internet. Patients need a health monitoring device to monitor their bodies continuously. With the help of this health monitoring device, accurate data of the patient's body can be collected anytime and anywhere. If the patient's body is in bad condition then the help of a mobile (which is connected to the IoT device via the internet) can be used to inform the doctor in a very easy way [21]. In IoT, interactions are made between the physical and digital worlds using sensors and actuators. In order to understand the physical parameters of an environment, sensors or networks of sensors are used. This processed sensor output is then sent to the main server or cloud via various network devices. Data can be accessed via the Internet from anywhere in the world. The main purpose of IoT technology is monitoring and control. Therefore, IoT-based monitoring is preferred over manual monitoring. The system is real-time monitoring of transformer parameters such as voltage, current, and temperature. This will help identify errors before a serious failure occurs [22]. With the advent of the novel Corona Virus, healthcare is now being given utmost importance in every country. So in this regard, an IoTbased health monitoring system is the best solution for this national epidemic. The Internet of Things (IoT) is the new revolution on the Internet, especially in the field of healthcare. With the increase in the use of wearable sensors and smartphones, remote healthcare monitoring has evolved at such a pace. IoT monitoring of health helps in preventing the spread of disease as well as to get a proper diagnosis of the state of health, even if the doctor is at a far distance. In this paper, a portable physiological is checking and framework is displayed, which can constantly screen the patient's heartbeat, temperature, and other basic parameters of the room [23]. In rural areas, due to lack of money, people cannot get their bodies checked properly and they do not get proper quality treatment. Many people get their treatment 
when they are in a very critical condition. The expensive cost of treatment cannot be afforded by many people in the village. So this type of projector has been considered to facilitate the treatment process. In developing countries, there is a lack of resources and management to reach the problems of individuals. An ordinary person is expensive for his health and cannot do daily check-ups. For this purpose, various systems have been created which make simple and secure care units. This system reduces time with handling tools safely [24]. Remote innovation has become much more popular in recent years because of the need to maintain different diversions. Most mechanical territories, particularly mechanization and control, have been taken over by IoT. With the help of IoT innovation, many medical clinics and well-being centers are opened. With the help of this astute framework, different limitations are seen that expand force, cost, and boost potency. The purpose of this paper is demonstrated by this brilliant framework. Specialists play a critical role in healthcare registration. A long time is required for registering, enrolling, and planning. The reports are generated later. Because of this extensive cycle, working individuals will in general disregard the exams or defer them. This vanguard approach reduces time utilization simultaneously. For many decades medical scientists have researched how to improve healthcare in a better way and give happiness to human lives [25]. Our project ensures the monitoring of an individual's heart rate and body temperature as well as other different kinds of illnesses like cholesterol, high blood pressure, low blood pressure, flu, etc. And in times of epidemics, the demand for healthcare monitoring systems has grown even more than before. Combining IoT with Arduino could be a new thing to introduce the Net of Things inpatient health care systems. Sensors sense the data with IoT-based wireless connectivity by using Arduino Uno and transmit the same to the IoT website. Some parameters like pulse rate, vital signs, pressure level, etc are tested by this projected PHMS system [26]. This device measures various parameters of the body like temperature, humidity, and pulse sensor, etc. The previous normal data is compared with the present data of the patient's body and if any abnormal data is found then that data is sent to the website of Thingspeak. After analyzing the data, if any abnormal data is found in the patient's body, a notification is sent to the doctor without any time loss through ThingSpeak [27]. This system has the ability to observe physiological parameters from the patient's body every 15 seconds and it can accumulate pulse, body temperature, and heartbeats all together from the patient's body and is responsible for transmitting data to the patient's health status stored in the cloud and using the WIFImodule to the IoT cloud platform. As a result of this proposed research, patients will get appropriate and effective health facilities [28]. The use of IoT facilities for health monitoring and fall detection can improve the daily lives of the elderly. The aim of this paper is to introduce an IoT-based system for monitoring health and detecting falls in the elderly. The main purpose of our work is to reduce the death rate of elderly people due to falling or lack of care. Our device will operate on the basis of threshold quality detected by the Arduino Uno R3 microcontroller [29]. In this project, using Arduino, sensors, and ESP 8266 wireless module we have created an IoT Based Patient Health Monitoring and Diagnosis system. For reserving and recovering the data we have applied it to the ThingSpeak IoT platform and API. ThingSpeak is an open-source IoT application and API to store and retrieve data. The present system can measure the pulse rate, body temperature, and blood pressure for these reason patients can communicate directly with physicians [30].

\section{Proposed Work:}

The world has become very conscious of its health since the pandemic began in 2019 [31]. Over the past two years, we have seen a shortage of medicines, hospital beds, and life-saving medical equipment. Two years into the pandemic and still we don't have enough necessary medical equipment like oximeters, BP monitors, and body temperature monitors. There are many people in India who still use analog thermometers, which are not quick and efficient, and also difficult for elderly people with limited vision. To solve all these problems we have come up with a very unique solution of providing all these monitoring devices under a single roof. Our Tetra Health Surveillance System offers BP monitoring, body temperature monitoring, $\mathrm{SpO} 2$ monitoring, and along with these individuals can also monitor the Temperature and Humidity of their surroundings using this device. It is an ideal solution for aged people who live by themselves or people who want to constantly monitor their vitals during this era of Covid-19 [32]. It eliminates the threat of constantly living in fear for your health. We have used four sensors in this device which are BP Sensor, Body Temperature Sensor, SpO2 Sensor, and a Temperature and Humidity sensor. Due to the fact that we have used the NODEMCU microcontroller in this device, this device becomes very cost-effective and can be produced in a large quantity. A user can easily monitor their health using this device and they can see the output on a computer or any smartphone that they have. The easy-to-use mechanism and cost-effectiveness make this a perfect fit for this pandemic era and also after the pandemic anyone can use this device to monitor their health very easily without going to a doctor. This device is also very portable too if you are traveling somewhere you can have this device in your backpack and can carry it with you. It is more convenient for people who are always on the go. It is an effective but portable solution for monitoring your health, which makes it different from other devices available in the market. This device also consumes very little power so you can also call it an energy-efficient device. All these features together make our Tetra Health Surveillance System a truly unique device to monitor your health [33].

\section{Flowchart of the Proposed System:}


In Figure 1, Microcontrollers and technology are improving every day. Technology is getting better and more sophisticated every day. In today's smart systems, microcontrollers play a vital role. Microcontrollers are at the heart of smart technologies. A single-chip processor is used to control machines or automate tasks in a very efficient and effective way [34]. Microcontrollers are in almost every device these days, whether it is a TV, air conditioning unit, vending machine, washing machine, etc.

You can think of a microcontroller as a portable computer because it has a CPU, timers, memory, input/output ports, and analog-digital converters all embedded in a single chip. The size of this mini-computer is very small and it is also very power efficient.

In our proposed system we have incorporated a microcontroller named "NodeMCU". Which acts as a central nervous system for our proposed system/device? The diagram above makes one thing really clear: we have four sensors that measure four different vital signs of a human body. In our system, we have got a blood pressure sensor, $\mathrm{SpO} 2$ sensor, body temperature sensor, and a temperature and humidity monitoring sensor. Three out of four of these sensors sense their data from a human body. It means these sensors need to come in direct contact with a human being and then only they can sense data. The other sensor which is the temperature and humidity sensor sense the data from the environment [35].

All four sensors are connected to the microcontroller in the middle and the sensor and send data to the microcontroller for processing. The microcontroller takes the data from all four of these sensors and processes each of them individually. The microcontroller takes very little time to process the inputs and due to the fact that this microcontroller has a WIFI module embedded in it, it can communicate with any other device that has WIFI. So using this feature we can display the output produced by the NodeMCU microcontroller on a computer or a mobile device. The output data is transferred to a mobile or computer via a wireless medium. In this way, with the help of the microcontroller all the sensors and the computer/mobile devices act as a single unit thus making our project "Tetra Health Surveillance System (THSS)".

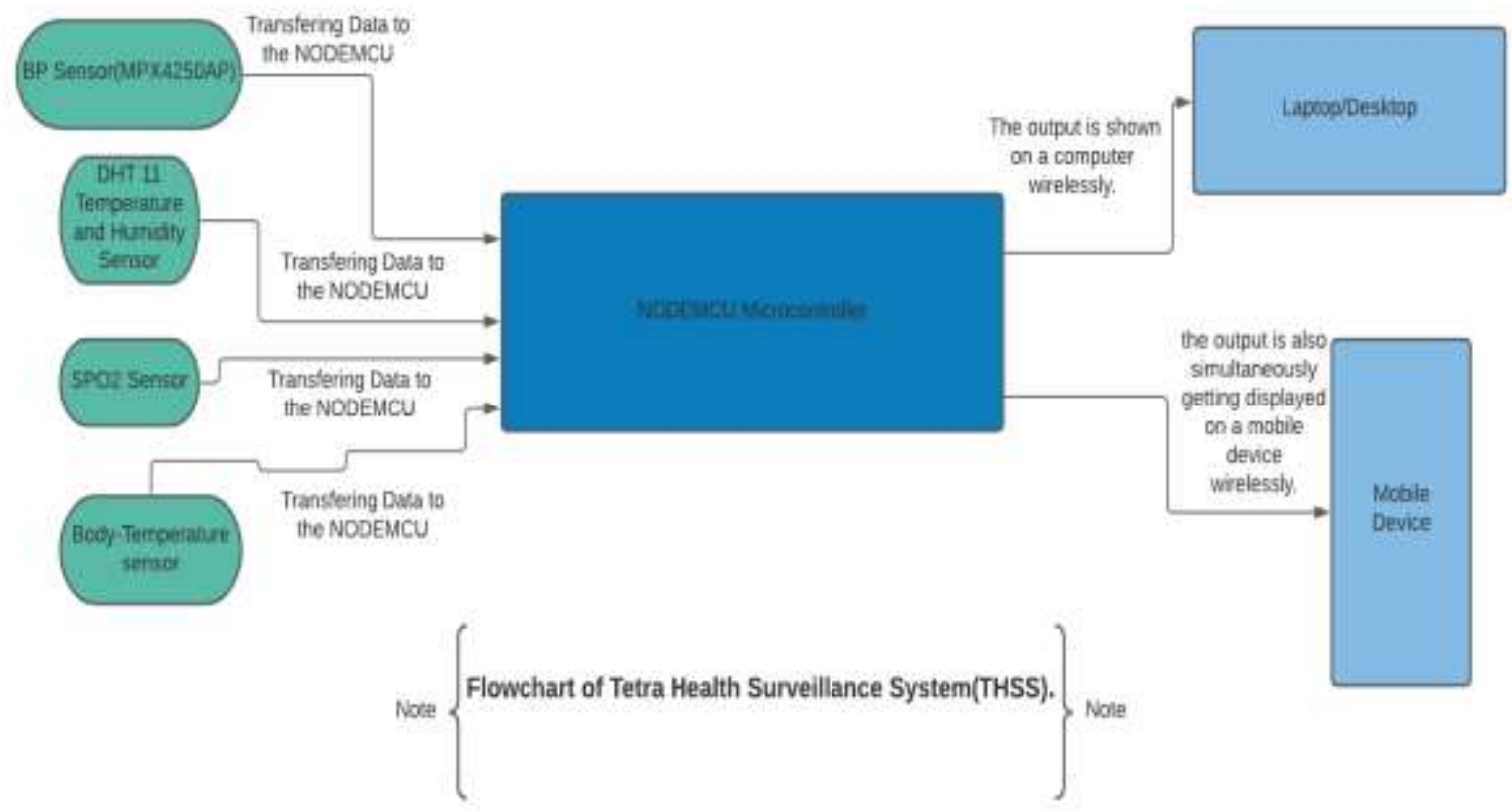

Figure 1 Structural Outline of Tetra Health Surveillance System (THSS)

\section{Functional Diagram:}

In Figure 2, the goal of this project is to monitor the human body using the device that we have made which is Tetra Health Surveillance System (THSS). From the functional diagram above you may already have an idea about how this system exactly works but here, we are going to discuss the diagram mentioned above in detail. We have used the following sensors for this project: BP Sensor (measures Human blood pressure), SpO2 Sensor (measures human blood oxygen saturation), Temperature Sensor (measures the amount of heat stored in the human body), and DHT11 Sensor (measures ambient air temperature and humidity) [36]. Among these sensors, the BP sensor, SpO2 sensor, and Body Temperature sensors are physical sensors that mean an individual has to come into contact with these sensors, and then they will work, while the DHT11 sensor works on the environment. In the diagram mentioned above, there are four sensors out of which three sensors (Body Temperature, SpO2, Blood Pressure) take measurements from a human body, and the other sensor which is DHT 11 sensor takes the input from the environment. We are using a single-board 
microcontroller called NodeMCU (Node Microcontrollers Unit is an open-source software and hardware development environment built around an inexpensive System-on-a-Chip (SoC)) to operate the output. The above-mentioned four sensors are all connected to this NodeMCU. RTOS (Real-Time Operating System) is supported on this microprocessor, which operates at a clock frequency of $80 \mathrm{Mhz}$ to $160 \mathrm{Mhz}$. For storing data and programs, NodeMCU has 128KB RAM and 4MB of Flash memory. With its high processing power and in-built Wi-Fi / Bluetooth capabilities, it is ideal for IoT projects. Through NodeMCU, with the help of the onboard WIFI, we can very easily display the output on a computer or a mobile device by just typing the IP address of the microcontroller in the address bar.

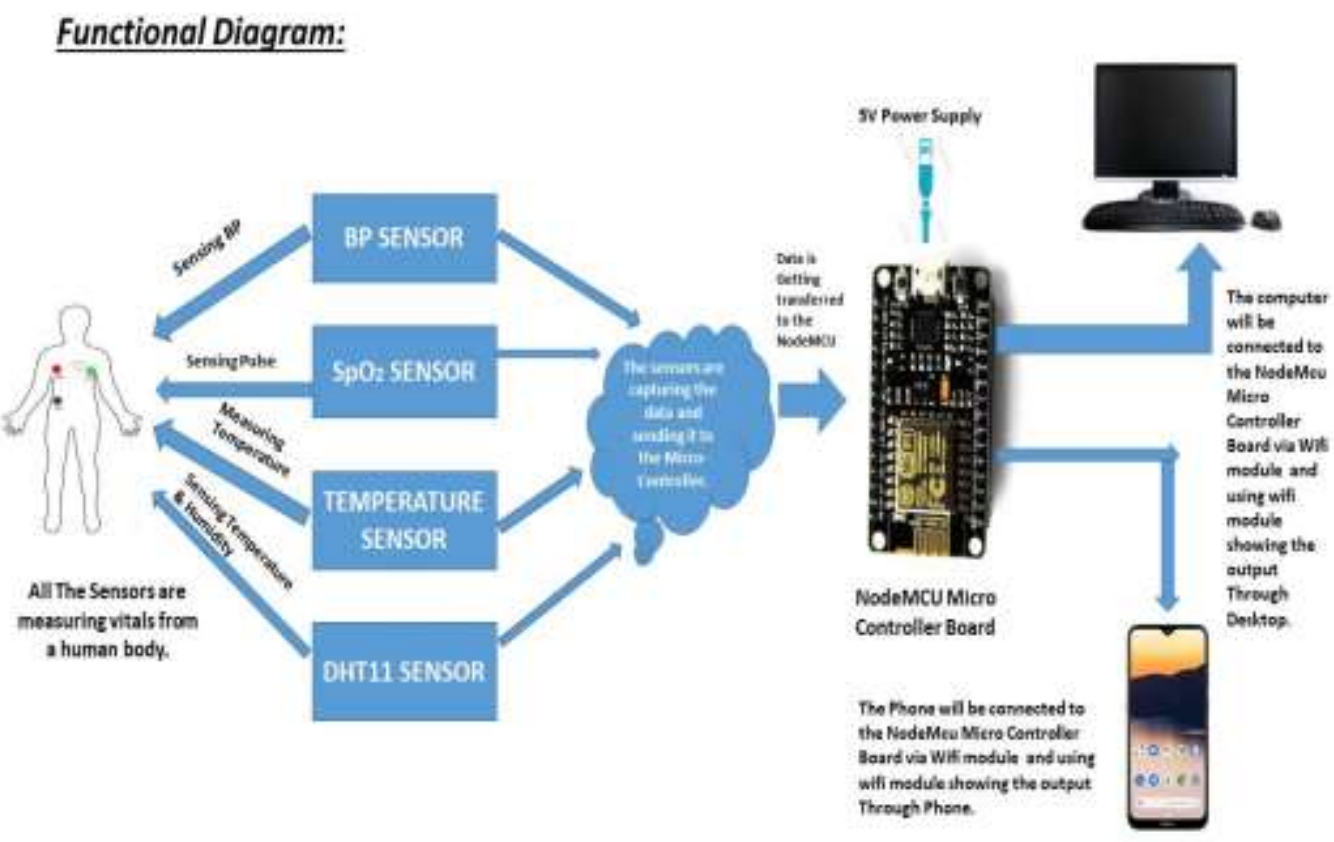

Figure 2 Performing Description of Tetra Health Surveillance System (THSS)

\section{Result \& Discussion/ Comparison with Current Product:}

The end product displays the body temperature, $\mathrm{SpO}$, heartbeat, temperature, and humidity of the room. The result can be viewed on a phone or computer using the ESPwebserver library for the NodeMCU microcontroller. This is the heart of our system. Tetra Health Surveillance offers reliable measures at an affordable price. Four sensors, all of which are very affordable, are used in this system, and the microcontroller is also very affordable, thereby bringing down the price. There are currently no systems that do not use either the Arduino microcontroller, the NodeMCU microcontroller, or the Raspberry Pi microcontroller [30,35]. There are very few systems on the market that display your body temperature, Room Temperature, and humidity, Heart Rate, and $\mathrm{SpO} 2$ level if you use an Arduino or Raspberry Pi microcontroller. Our system displays all these vital signs on one screen, and it takes only a few seconds for the user to see the output on their mobile or computer. Below we will explain the working of our Tetra Health Surveillance System.

A switch embedded in the system is used to turn on the system. When the system is turned on, it automatically connects to your WIFI/Mobile Hotspot. A rechargeable battery powers this device. Additionally, if the battery runs out, you can connect this device to any power bank or external power source and it will work fine.

Next, the user needs to put their finger in the SpO2/BMP sensor which will measure their Heart Beat and SpO2 level. On the other side, the user needs to hold the body temperature sensor in their hand so that it can detect their body temperature in real-time. The room Temperature and Humidity sensor collects data from the environment. The user needs to copy the IP address displayed on the digital display on our device and put it into the browser on their computer or mobile phone [36]. Figure 3 has been shown the basic circuit model of Tetra Health Surveillance System (THSS). 




Figure 3 Circuit model of Tetra Health Surveillance System (THSS)

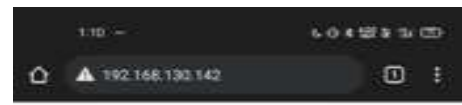

\section{Tetra Health \\ Surevillance \\ System}

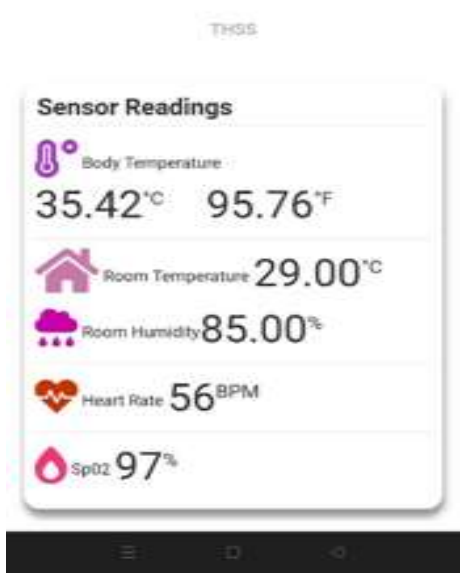

Figure 4 Output of Tetra Health Surveillance System (THSS)

After entering the IP on your browser the real-time output can be seen on your Mobile Phone as shown in figure 4 . Other systems on the market are very complicated and not everyone is able to grasp their complex architectures [37, 38]. Tetra Health Surveillance is different from other systems on the market due to its ease of use and the financial feasibility of producing these systems in large quantities. Our system is also very economical and provides all the vital readings you need in this digital age at an affordable price. The other major difference between this system and other existing systems is that our research has shown that other systems available in the market don't provide accurate or reliable data. We provide accurate data within a short timeframe. 6.1 explained in the below algorithm.

\section{1 Operational Algorithm:}

1) Turn on the system.

2) Connect the system with the internet.

3) Connecting the sensors with our bodies.

a) $\mathrm{BP} \& \mathrm{SpO} 2$ sensors.

b) Physical Temperature sensor. 
Both the sensor's data varies from person to person.

4) Another sensor (DHT11) is attached to the system which automatically senses the indoor humidity and temperature.

5) After sensing, the real-time data will be generated and processed through NodeMCU.

6) An IP address is displayed in the $16 \times 2$ display.

7) Open a preferred browser and give that IP in the search bar to see the information.

8) A 16x2 display is attached to the system to show the same information.

9) If the data is not picking up accurately, we have to reset the whole system with the reset button present in the system then,

go back to 2 and repeat the process once again.

10) Turn off the system.

\section{Conclusion:}

This is a simple IoT-based health monitoring system that can measure, display, and store basic health parameters like body temperature, heart rate, and oxygen saturation. The tests can be done outside of the hospital, and medical staff can still track the results in real-time. During crises, nurses and doctors might find the system useful, as raw medical data can be analysed in a short amount of time. It will improve the current healthcare system and could save many lives. When the caregivers are absent, this is very useful, especially when caring for elderly patients or children. Utilizing an IoT-based mobile app can significantly reduce the hospital's administration costs, lowering the patient's treatment costs, and making healthcare more affordable. Monitoring medical patients continuously is necessary due to their importance. By using the desktop and mobile message service, we are able to monitor patients continuously. The paper also compared the early-aged medical system with modern health monitoring. It involves reducing health care costs and time, especially for people living in rural areas.

\section{References}

2. Ahona Ghosh, Sandip Roy, Haraprasad Mondal, Suparna Biswas, \& Rajesh Bose (2021). Mathematical modelling for decision making of lockdown during COVID-19. Applied Intelligence, 1-17.

3. Arkadeep Nandi, Sandipan Basu, Abhisek Kumar, Prajoy Dutta (2021). IOT/CLOUD-BASED HEALTH MONITORING SYSTEM (TEMP, PULSE RATE, SpO2). RCC INSTITUTE OF INFORMATION TECHNOLOGY, CANAL SOUTH ROAD, BELIAGHATA, KOLKATA - 700015, WEST BENGAL, Maulana Abul Kalam Azad University of Technology (MAKAUT).

4. Sonam Gupta, Lipika Goel, Abhay Kumar Agarwal (2021) A Novel Framework of Health Monitoring Systems. International Journal of Big Data and Analytics in Healthcare, Volume 6 • Issue 1 • January-June 2021.

5. Indranil Sarkar, Bijoy Pal, Arnab Datta, Sandip Roy (2020). Wi-Fi-based portable weather station for monitoring temperature, relative humidity, pressure, precipitation, wind speed, and direction. In Information and Communication Technology for Sustainable Development (pp. 399-404). Springer, Singapore.

6. K.V. Sowmya, V. Teju (2021) AN EFFICIENT HEALTH MONITORING SYSTEM WITH TEMPERATURE AND HEART RATE SENSORS USING IoT. European Journal of Molecular \& Clinical Medicine ISSN 25158260 Volume 08, Issue 2, 2021.

7. Sanjay Balwani, Anil Bavaskar, Irfan Javed, Sarfraz Ali (2020) An IoT-based Healthcare for Remote Patient Monitoring System. Annals of R.S.C.B., ISSN: 1583-6258, Vol. 24, Issue 2, 2020.

8. Ms. Yedavally Shivani, Ms. Ravulapati Dharani, Ms. Aruva Neelima, Dr. M. Varaprasad Rao (2020) PATIENT HEALTH MONITORING WITH DOCTOR ALERT REPORTING OVER IOT. Journal of Interdisciplinary Cycle Research Volume XII, Issue IV, April/2020 ISSN NO: 0022-1945 Volume XII, Issue IV, April/2020.

9. G. Joga Rao, P. Karthik, G. Sai Sameer Kumar, N. Ajay, K. Anil Kumar, J. Durga Prasad(2020) Health Monitoring System of Patients Using IoT. International Journal of Scientific Research in Science, Engineering, and Technology ( 2020 IJSRSET | Volume 7 | Issue 2 | Print ISSN: 2395-1990 | Online ISSN: 2394-4099.

10. Shashidhar R, Abhilash S, Sahana V, Alok N A, Roopa M (2020) Iot Cloud: In Health Monitoring System INTERNATIONAL JOURNAL OF SCIENTIFIC \& TECHNOLOGY RESEARCH VOLUME 9, ISSUE 01, JANUARY 2020 ISSN 2277-8616.

11. Md. Abbas Ali Khan, Md. Sadekur Rahman (2019) IoT-BASED PULSE RATE AND BODY TEMPERATURE MONITORING SYSTEM.DAFFODIL INTERNATIONAL UNIVERSITY DHAKA, BANGLADESH DECEMBER 2019.

12. LIM CHEE YUAN (2019) IoT BASED HEALTH MONITORING SYSTEM Lee Kong Chian Faculty of Engineering and Science University Tunku Abdul Rahman. 
13. Biju Rajan B1, Amanraj S2, Akhil S3, Nayana S41 Assistant Professor, Dept. of EEE, VKCET, Thiruvananthapuram.2,3,4, Student, Dept. of EEE, VKCET, Thiruvananthapuram(2019).IoT based Distribution Transformer Health Monitoring System using Arduino, Nodemcu and Thingspeak.

14. Fahim Faisal, Syed Akhter Hossain (2019), IoT Based Remote Medical Diagnosis System Using NodeMCU Dept. of Computer Science and Engineering Daffodil International University Dhaka, Bangladesh.

15. Md. Sharif Hasnat, Shamim-Al-Mamun, Fariha Hossain, Sumaiya Hossain (2019), United International University Dhaka, Bangladesh February, 2019

16. Khin Thet Wai, Nyan Phyo Aung, Lwin Htay (2019), Internet of Things (IoT) Based Healthcare Monitoring System using NodeMCU and Arduino UNO Khin Thet Wai, Nyan Phyo Aung, Lwin Htay Department of Electronic Engineering, Technological University, Mandalay, Myanmar.

17. Md. Raseduzzaman Ruman, Khan Roushan Jahan, Amit Barua, Md. Jamil Roni, Waladur Rahman(2020),IoT Based Emergency Health Monitoring System,2020 International Conference on Industry 4.0 Technology (I4Tech) Vishwakarma Institute of Technology, Pune, India. Feb 13-15, 2020.

18. Ramesh Saha1, Suparna Biswas2 - Satyajit Sarmah, Sushanta Karmakar3,Pranesh Das1 A Working Prototype Using DS18B20 Temperature Sensor and Arduino for Health Monitoring Received: 12 December 2020 / Accepted: 16 December 2020 / Published online: 12 January 2021 The Author(s), under exclusive licence to Springer Nature Singapore Pte Ltd. part of Springer Nature 2021.

19. Salam khalaf Abdullah1(2019), Remote Heart Rate Monitor System using NodeMCU Microcontroller and easy pulse sensor v1.1, Institute of Technology-Baghdad, Middle Technical University, Baghdad, Iraq Email: hassan19851988@yahoo.com (Alhassan H Ismail).

20. TEJ PRAKASH SAHU1, DR. VINAY KUMAR JAIN2(2020), IoT based Patient Monitoring System 1(ME, Student) Department of Electronics \& Telecommunication Engineering, Shri Shankaracharya Group of Institutions SSTC Junwani Bhilai (CG) 2Asso. Professor \& Head of Department of Electronics and Telecommunication Shri Shankaracharya Group of Institutions SSTC Junwani Bhilai (CG).

21. Prasun Biswas1, Shreyashi Haldar2(2020), Remote Health Monitoring System using Internet of Things,UG Student, Department of Electrical Engineering, RCC Institute of Information Technology, MAKAUT, Kolkata, INDIA.

22. Khin Thet Wai, Nyan Phyo Aung, Lwin Lwin Htay, Internet of Things (IoT) Based Healthcare Monitoring System using NodeMCU and Arduino UNO, Department of Electronic Engineering, Technological University, Mandalay, Myanmar, e-ISSN: 2456 - 6470, Volume 3 Issue 5, August 2019.

23. Biju Rajan B, Amanraj S, Akhil S, Nayana S, IoT based Distribution Transformer Health Monitoring System using Arduino, Nodemcu and Thingspeak, Assistant Professor, Dept. of EEE, VKCET, Thiruvananthapuram.Student, Dept. of EEE, VKCET, Thiruvananthapuram, ISSN: 2278-0181, Vol. 8 Issue 04, April-2019.

24. Prajoona Valsalan, Tariq Ahmed Barham Baomar, Ali Hussain Omar Baabood, IOT BASED HEALTH MONITORING SYSTEM, Department of Electrical and Computer Engineering, Dhofar University, Salalah, Sultanate of Oman, ISSN- 2394-5125, Vol 7, Issue 4, 2020.

25. Shivkumar Dharmoji, Akshata Anigolkar, Prof. Shraddha M, IoT based Patient Health Monitoring using ESP8266, Student, Electronics and Communication Department, Jain College of Engineering, Belagavi, India, eISSN: 2395-0056, Volume: 07 Issue: 03, Mar 2020.

26. MANDUVA SIRI CHANDANA, Dr.M.R.ARUN, An IoT based Patient Health Monitoring System using Node MCU, M.Tech Scholar, Dept. Of ECE, PBR Visvodaya Institute of Technology\& Science Kavali, SPSR Nellore (D.T), A.P, e-ISSN: 2395-0056, Volume: 07 Issue: 10 ,Oct 2020.

27. Richa, Anwesha Das, Ajeet Kumar Kushwaha, Prof. Mini Sreejeth, An IoT based Health Monitoring System using Arduino Uno, Department of Electrical Engineering, Delhi Technology University, New Delhi, ISSN: 22780181, Vol. 10 Issue 03, March-2021.

28. Sudha Senthilkumar, Brindha, Abishek Kumar, Patients Health monitoring System using IOT, School of Information Technology and Engineering, Vellore Institute of Technology, Vellore, Tamil Nadu, India, Vol.10, No.4, April-2019.

29. Md. Raseduzzaman Ruman, Khan Roushan Jahan, Amit Barua, Md. Jamil Roni, Waladur Rahman, Md. Foyjur Rahman, IoT Based Emergency Health Monitoring System, Electrical \& Electronic Engineering American International University- Bangladesh, Dhaka, Feb 13-15, 2020.

30. Noushin Jannal, M. tanzeeb Rubaiat Haque, IOT Based Health Monitoring \& Fall Detection System, International Journal Of Scientific \& Engineering Research, ISSN 2229-5518, Volume 10, Issue 6, June-2019.

31. P. Jebane, P. Anusuya, M.Suganya, S. Meena, M. Diana amutha priya, IOT BASED HEALTH MONITORING AND ANALYSING SYSTEM USING THINGSPEAK CLOUD \& ARDUINO, Electronics and Communication Engineering, Fatima Michael College of engineering and technology, Madurai, India, ISSN NO 2582-0958, Volume 5, Issue 4, August 2021. 
32. Banashree Debnath, Rajesh Dey, \& Sandip Roy (2019, February). Smart switching system using bluetooth technology. In 2019 Amity International Conference on Artificial Intelligence (AICAI) (pp. 760-763). IEEE.

33. Roy, S., Biswas, S. P., Mahata, S., \& Bose, R. (2018, October). Time series forecasting using exponential smoothing to predict the major atmospheric pollutants. In 2018 International Conference on Advances in Computing, Communication Control and Networking (ICACCCN) (pp. 679-684). IEEE.

34. Bose, R., Chakraborty, S., \& Roy, S. (2019, February). Explaining the workings principle of cloud-based multifactor authentication architecture on banking sectors. In 2019 Amity International Conference on Artificial Intelligence (AICAI) (pp. 764-768). IEEE.

35. Bose, R., Roy, S., Mondal, H., Chowdhury, D. R., \& Chakraborty, S. (2021). Energy-efficient approach to lower the carbon emissions of data centers. Computing, 1-19.

36. Bose, R., Dey, R. K., Roy, S., \& Sarddar, D. (2020). Time series forecasting using double exponential smoothing for predicting the major ambient air pollutants. In Information and communication technology for sustainable development (pp. 603-613). Springer, Singapore.

37. Roy, S., Bose, R., \& Sarddar, D. (2017). Smart and healthy city protecting from carcinogenic pollutants. Int. J. Appl. Environ. Sci, 12, 1661-1692.

38. Bose, R., Saha, M. K., \& Sarddar, D. (2015). Fog computing made easy with the help of Citrix and Billboard manager. International Journal of Computer Applications, 121(7), 19-23.

39. Roy, S., Sen, A., Roy, T., \& Sarddar, D. (2015). Energy Efficient WMSN for Virtual Sensor-Based Global Information Sharing using Mobile Cloud. International Journal of Scientific \& Engineering Research, 6(6), 1127. 